atteints (32 standards et 12 miniatures) en utilisant un questionnaire disponible sur un site internet où nous décrivions la maladie. Tous les chiens inclus dans l'étude ont subis un examen clinique, neurologique et comportemental et ont été suivis durant une période de 2 ans. Nous décrivons ainsi une maladie très pléiomorphe mais unique au sein de laquelle ces comportements de " spinning " ou de " tail-chasing " coexistent et peuvent s'exprimer de façon occasionnelle et durer de courts instants mais peuvent également durer plusieurs heures avec une totale dissociation du chien de son environnement. Des comportements hallucinatoires ou de type psychotiques (phases de fixité, crises d'agression ou grognements sans raisons ou non dirigés et dans le vide ou dirigés vers des objets inanimés, gobage de mouches imaginaires, léchage de surface ... ) ont été rapportés dans la majorité des cas (31 chiens). La maladie apparaît le plus souvent avant 7 mois (35 chiens), l'âge médian est de 5 mois, il n'y a pas de prédisposition sexuelle. Nous décrivons 4 stades de maladie qui en reflètent la gravité et l'évolution des chiens au sein de ces différents stades. 14 chiens ont été euthanasiés du fait de la gravité des symptômes. Des bilans biochimiques exhaustifs, des examens d'imagerie par résonnance magnétique de l'encéphale, et des examens histologiques de l'encéphale ont été réalisés sur une sélection de chiens et n'ont pas montré d'anomalies. Un traitement à base de phénobarbital et de bromure de potassium réalisé sur 27 chiens a permis de contrôler la maladie dans 8 cas et d'améliorer les symptômes dans 11 cas. Afin de réaliser des analyses génétiques, deux pédigrées (BT standard et miniature) ont été construits et nous ont permis de proposer un mode de transmission autosomal récessif. En utilisant des échantillons sanguins de nos chiens français couplés avec des prélèvements de chiens finlandais, nous avons regardé si le locus CFA7 (et en particulier le gène $\mathrm{CDH} 2$ ) associé au comportement compulsif de suçage de flanc chez le doberman été associé au spinning du BT, ce qui n'est pas le cas. Nous avons également réalisé une étude d'association " whole genome " (Illumina 200K SNP arrays) qui à ce jour n'est pas concluante et ne permet pas de proposer de gène(s) candidat(s). Nous concluons que le tournis (S ou TC) chez le BT constitue indubitablement le symptôme d'une maladie neurologique évolutive et pléiomorphe. La réponse au traitement anticonvulsivant laisse suspecter une forme d'épilepsie se présentant sous la forme de crises convulsives partielles complexes mais la pathogénie précise de la maladie reste à déterminer. Certains des symptômes peuvent être l'expression d'un état psychotique post-ictal ou périictal mais la coexistence de troubles compulsifs avec des crises convulsives au sein d'une affection neurologique complexe comme l'autisme doit être envisagé.

Mots clés : comportements de tournis; comportements de chasser sa queue; Troubles Obsessionnels Compulsifs ; chien ; épilepsie

\title{
CONDOG PROJECT: INTEGRATED TEMPERAMENT ASSESSMENT TO CONSTRUCT A TOOL FOR EARLY PREDICTION OF BEHAVIORAL PROBLEMS
}

C.M.M. Lensen ${ }^{1,}{ }^{*}$, C.P.H. Moons ${ }^{2}$, C. Diederich ${ }^{1}$,

${ }^{1}$ University of Namur, Department of veterinary medicine (IVRU), rue de Bruxelles 61, 5000 Namur, BELGIUM

${ }^{2}$ Ghent University, Department of Nutrition, Genetics and Ethology, Faculty of Veterinary Medicine, Heidestraat 19, 9820 Merelbeke, BELGIUM

*Corresponding author: clensen@fundp.ac.be

Numerous dogs live in our society, of which several develop behavioral problems. These dogs can pose a problem to other dogs and humans and they are often abandoned, sent to re-homing centers or euthanized. An early prediction of problem behavior could prevent these problems to occur. In the CONDOG project the development of dog temperament is studied in order to identify at an early stage individuals that are susceptible of developing behavioral problems. Domestic dogs of four breed clusters and two control groups (laboratory and aggressive dogs) are repeatedly evaluated (ages: 6, 12, 18 months) in the form of behavioral tests (reactivity), physiological measurements (heart rate variability, salivary markers), and owners' evaluations of their dogs' behavior to identify 
potential behavioral problems (validated C-BARQ questionnaire). In addition, veterinary practitioners assess the dogs' behavior during physical examination, and at an age of 18 months all dogs will undergo a standardized test of fear and aggression (SAB test). By comparing these different assessments with the occurrence of behavioral problems, an optimal age for identifying an animal with (future) problems, as well as the parameters that validate this prediction are identified. Based on these findings, a simplified tool for evaluating temperament will be proposed to veterinary practitioners, so that they can inform their clients and together with them take measures to prevent behavioral problems.

Key words: temperament assessment; behavior problem; dog; prediction

Research funded by the Federal Public Service Health, Food Chain Safety and Environment (contract CONDOG RT 10/12), with the support of Royal Canin S.A.

PROJET CONDOG: ÉVALUATION INTÉGRÉE DU TEMPERAMENT POUR CONSTRUIRE UN OUTIL DE PRÉDICTION DES PROBLÈMES COMPORTEMENTAUX

C.M.M. Lensen ${ }^{1},{ }^{*}$, C.P.H. Moons ${ }^{2}$, C. Diederich ${ }^{1}$,

${ }^{1}$ Facultés Universitaires Notre-Dame de la Paix, Département de Médecine Vétérinaire (URVI), rue de Bruxelles 61, 5000 Namur, BELGIQUE

${ }^{2}$ Universiteit Gent, Vakgroep Voeding, Genetica en Ethologie, Faculteit Diergeneeskunde, Heidestraat 19, 9820 Merelbeke, BELGIQUE

*Correspondant: clensen@fundp.ac.be

De nombreux chiens vivent dans notre société, dont plusieurs développent des problèmes comportementaux. Ils deviennent alors un problème pour les autres chiens et pour les humains. Ils sont souvent abandonnés, envoyés dans des refuges ou euthanasiés. Une prédiction précoce des problèmes de comportement pourrait éviter qu'ils ne se produisent. Le projet CONDOG étudie le développement du tempérament chez le chien afin d'identifier de manière précoce les individus susceptibles de développer des problèmes de comportement. Les chiens domestiques des quatre groupes de race et de deux groupes de contrôle (chiens de laboratoire et chiens agressifs) seront évalués à plusieurs reprises (âge: 6, 12, 18 mois) à l'aide de tests comportementaux (réactivité), de mesures physiologiques (variabilité du rythme cardiaque, des marqueurs salivaires) et d'un questionnaire aux propriétaires afin d'identifier des problèmes potentiels de leur chien (le questionnaire validé C-BARQ). En outre, des vétérinaires praticiens évalueront le comportement des animaux en clinique, et à 18 mois, tous les chiens seront soumis à un test standardisé de la peur et l'agression ( $S A B$ test). En comparant ces différentes évaluations avec l'apparition de problèmes comportementaux, l'identification d'un âge optimal pour mettre en évidence un animal à problème (futur) ainsi que les paramètres qui valident cette prédiction seront mis en œuvre. Basé sur ces résultats, un outil simplifié d'évaluation du tempérament pourra être proposé aux vétérinaires praticiens afin qu'ils puissent en informer leurs clients et avec eux, mettre en place des mesures de prévention des problèmes comportementaux adaptées.

Mots clés: évaluation du tempérament; problème comportemental; chien; prédiction

Recherche financée par le SPF Santé publique, Sécurité de la Chaîne alimentaire et Environnement (contrat CONDOG RT 10/12), avec le soutien de Royal Canin sa. 